Proceedings

\title{
Comparison of Untapped Agroindustrial Olive Resources with Olive Leaves ${ }^{\dagger}$
}

\author{
María del Mar Contreras 1,2,*, Irene Gómez-Cruz 1,2, Inmaculada Romero 1,2 and Eulogio Castro 1,2 \\ 1 Department of Chemical, Environmental and Materials Engineering, Universidad de Jaén (UJA), \\ 23071 Jaén, Spain; igcruz@ujaen.es (I.G.-C.); iromero@ujaen.es (I.R.); ecastro@ujaen.es (E.C.) \\ 2 Centre for Advanced Studies in Earth Sciences, Energy and Environment (CEACTEMA), UJA, \\ 23071 Jaén, Spain \\ * Correspondence: mcgamez@ujaen.es \\ + Presented at the 1st International Electronic Conference on Biomolecules: Natural and Bio-Inspired \\ Therapeutics for Human Diseases, 1-13 December 2020; Available online: https://iecbm2020.sciforum.net/.
}

Citation: Contreras, M.d.M.;

Gómez-Cruz, I.; Romero, I.; Castro, E. Comparison of Untapped

Agroindustrial Olive Resources with Olive Leaves. Proceedings 2021, 79, 3. https://doi.org/10.3390/IECBM2020-0 8585

Published: 30 November 2020

Publisher's Note: MDPI stays neutral with regard to jurisdictional claims in published maps and institutional affiliations.

Copyright: $\subset 2020$ by the authors. Licensee MDPI, Basel, Switzerland. This article is an open access article distributed under the terms and conditions of the Creative Commons Attribution (CC BY) license (http://creativecommons.org/licenses /by/4.0/).

\begin{abstract}
Olive leaves are a source of valuable compounds with applications in the phytopharmacy sector, among others. Nevertheless, there are untapped olive resources that can give new clues for the discovery of natural bioactive compounds. Therefore, in this study the antioxidant composition of exhausted olive pomace (EOP) and a new byproduct, the residual fraction from olive pit cleaning (RFOPC), was characterized and compared to olive leaves, which have been extensively studied as a source of bioactive compounds. The chemical characterization showed that all of these byproducts contain high amounts of extractives; in all cases it was higher than $26 \%$. This is interesting since the extractive fraction contains non-structural compounds, including phenolic compounds and triterpenic acids. Then, ultrasound-assisted extraction was applied to recover phenolic compounds from this fraction using an aqueous-ethanolic solution. All the extracts showed antioxidant properties and the total phenolic content ranged from 69 (RFOPC) to 140 (EOP) g of gallic acid equivalents $/ \mathrm{kg}$ dry weight. The profile obtained by liquid chromatography-mass spectrometry was different, suggesting some chemical differences in the phenolic composition. Moreover, some triterpenic acids were characterized, which suggest that multifunctional ingredients can be obtained.
\end{abstract}

Keywords: agro-industrial waste; exhausted olive pomace; hydroxytyrosol; multifunctional extracts; olive leaves; triterpenic acids

\section{Introduction}

Besides olive oil, olive leaf and fruit extracts are currently applied as key ingredients of nutraceuticals and dietary supplements due to their cardiovascular health promoting properties, among other effects. These extracts contain phenolic compounds, like hydroxytyrosol and their derivatives, e.g., oleuropein, hydroxytyrosol glucoside and/or verbascoside [1-3], which have shown therapeutic potential in in vitro, in vivo and clinical studies [4-6]. Morevoer, hydroxytyrosol consumption seems to be safe, and not genotoxic or mutagenic in vitro [7]. The hydroxytyrosol cluster varies in composition, qualitatively and quantitatively, depending on the olive product, cultivar, origin, pedoclimatic conditions, and extraction method, among other factors [1-3,8,9].

Today, the olive oil industry generates a huge amount of byproducts/wastes, consisting of leafy and defatted fruit residues, i.e., olive mill leaves and olive pomace. In Spain, olive pits are partially recovered from the olive pomace, and are used as biofuel to produce heat [10]. Moreover, the cleaning of this byproduct to enhance their fuel properties and the extraction of the residual oil in the olive pomace generates other wastes known as residual fraction from olive pit cleaning (RFOPC), or pulp, and exhausted olive pomace (EOP), respectively [3]. The characterization of these sources for the production 
of bioactive components is an important step toward sustainable development and it is also a new way to find new potential sources of hydroxytyrosol derivatives. Therefore, in this work, olive leafy biomass, EOP, and RFOPC have been compared as antioxidant sources using colorimetric assays and high-performance liquid chromatography analyses.

\section{Material and Methods}

\subsection{Olive-Derived Biomasses and Chemical Characterization}

Olive-derived biomasses were supplied by local companies in Jaén (Andalusia, Spain). Olive leaves were obtained in June 2019, also in this region. They had moisture levels up to $10 \%$ and were milled before analysis and extraction using an Ultra Centrifugal Mill ZM 200 (Retsch GmbH, Haan, Germany).

The chemical composition of the samples was determined according to the standard procedure from the National Renewable Energy Laboratory (NREL). This included a previous two-step extraction procedure with water and ethanol to determine the content of extractives and the analysis of lignin, carbohydrates, and ash [11]. Moreover, the protein content was estimated by considering the nitrogen content as determined by elemental analysis (TruSpec Micro, Leco, St. Joseph, MI, USA) and using a conversion factor of 6.25 .

\subsection{Extraction Method}

The samples were extracted using an aqueous ethanol solution $(47 \%, v / v)$ at $6 \%(w / v)$ for 50 min using an ultrasonic bath (Ultrasons, J.P. Selecta, Barcelona, Spain) [12]. After centrifugation, the supernatants were collected and filtered before analysis (nylon filters of $0.45 \mu \mathrm{m}$ pore size) (SinerLab Group, Madrid, Spain).

\subsection{Measurement of the Total Phenol Content and Antioxidant Capacity}

The determination of the total phenol content (TPC), based on the Folin-Ciocalteu method and the $\operatorname{ABTS}^{\mathrm{TM}}$ (2,2'-azino-bis(3-ethylbenzothiazoline-6-sulfonic acid) diammonium salt) radical scavenging assay, were determined according to Medfai et al. [8] in a Bio-Rad iMarkTM microplate absorbance reader (Hercules, CA, USA) at $750 \mathrm{~nm}$. The TPC results were expressed as gallic acid equivalents (GAE) using a curve of gallic acid from 25 to $300 \mu \mathrm{g} / \mathrm{mL}$. Moreover, the ABTS results were expressed as Trolox equivalents (TE) using a curve of Trolox from 6 to $330 \mu \mathrm{M}$.

\subsection{Reversed-Phase High Performance Liquid Chromatography Analyses}

Reversed-phase (RP)-high performance liquid chromatography (HPLC) analyses were performed using a Shimadzu Prominence UFLC chromatograph (Kyoto, Japan) equipped with a diode array detector (DAD). The column used was a C18 BDS HYPERSIL column $(250 \mathrm{~mm} \times 4.6 \mathrm{~mm}, 5 \mu \mathrm{m})$ (Thermo Fisher Scientific Inc., Waltham, Massachusetts, USA). A ternary gradient was applied using an aqueous solution of orthophosphoric acid $(0.2 \%, \mathrm{v} / \mathrm{v})$, methanol, and acetonitrile according to Gómez-Cruz et al. [13]. The elution flow was $1 \mathrm{~mL} / \mathrm{min}$, the oven temperature was set at $30^{\circ} \mathrm{C}$, and the volume of sample injected was $20 \mu \mathrm{L}$. Hydroxytyrosol, luteolin 7-O-glucoside, and oleuropein were quantified at $280 \mathrm{~nm}$ by comparison with external calibration curves obtained using their commercial standards. Similarly, maslinic and oleanolic acids were quantified at $210 \mathrm{~nm}$. All the standards were supplied by Extrasynthese (Genay, France).

Additionally, accurate mass analyses were performed using an HPLC (Agilent 1200) coupled to quadrupole-time-of-flight (QTOF)-MS and MS/MS (Agilent 6530B) via electrospray ionization according to Ammar et al. [14]. 


\section{Results and Discussion}

\subsection{Chemical Composition and Antioxidant Characteristics}

The chemical characterization showed that all these byproducts contain high amounts of extractives; in all cases the content was higher than $26 \%$. Particularly, it was the major component in OL and EOP. Since this fraction contains bioactive compounds [3], it seems reasonable to explore it. Therefore, the extracts obtained by ultrasound-assisted extraction (UAE) were evaluated in terms of TPC and antioxidant activity using an aqueous ethanol solution as a food/pharma grade solvent. The results are shown in Figure 1. OL has the highest TPC value, but the EOP extract showed the highest antioxidant properties. Since there was no correlation $(r=0.452)$, it is expected that the phenolic profiles were different.

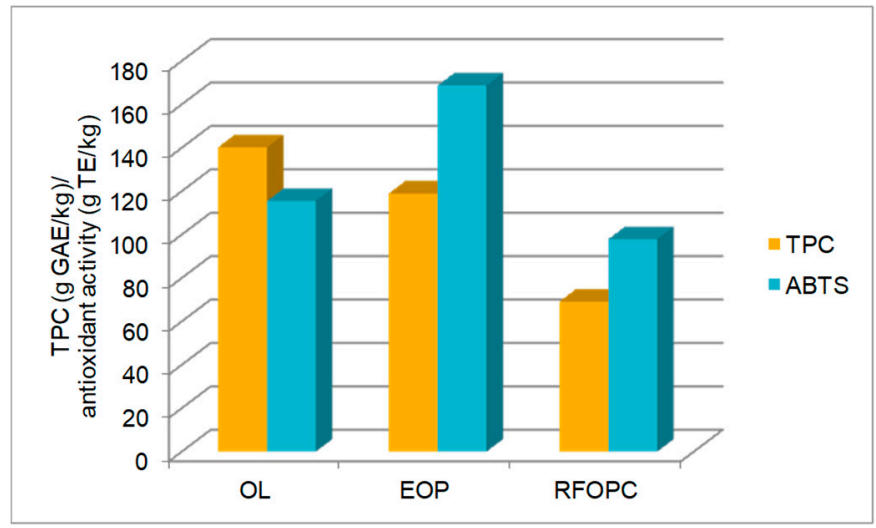

Figure 1. Total phenolic content (TPC) and antioxidant activity determined by the ABTS ${ }^{\mathrm{TM}}$ assay of aqueous ethanol extracts from of olive-derived biomasses: OL (olive leaves), EOP (exhausted olive pomace), and RFOPC (residual fraction from olive pit cleaning).

\subsection{HPLC Profiles and Quantitative Analysis}

The liquid extracts were analyzed by RP-HPLC-DAD to compare their phenolic profiles. Figure 2 shows the chromatograms at $280 \mathrm{~nm}$ and the peaks corresponding to hydroxytyrosol, luteolin 7-O-glucoside, and oleuropein. All these compounds were present in OL and EOP, while only hydroxytyrosol was detected in RFOPC. The presence of oleuropein was clearly high in OL, while hydroxytyrosol was high in EOP, as the intensity of the peaks was higher in their corresponding chromatograms. Nonetheless, the total solid extraction yield was low in RFOPC, and so the richness in hydroxytyrosol is enhanced in the dry extract.

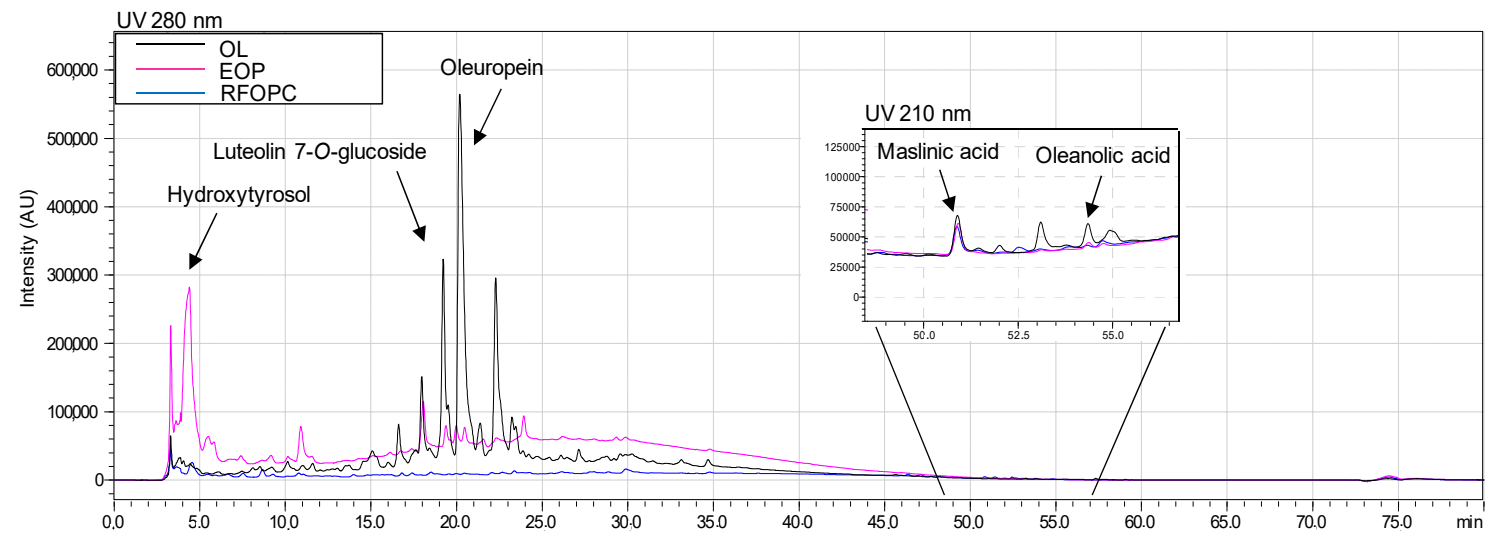

Figure 2. Chromatograms obtained at 280 and $210 \mathrm{~nm}$ of the aqueous ethanolic extracts of olive leaves (OL), exhausted olive pomace (EOP), and residual fraction from olive pit cleaning (RFOPC). 
Moreover, Figure 3a depicts the concentration of these compounds in OL, EOP, and RFOPC extracts. This highlights that, besides olive pomace [3], EOP is a rich source of hydroxytyrosol, which can, in part, promote the antioxidant activity of the extract. This was the only phenolic compound whose content showed certain positive correlation with the antioxidant activity $(r=0.862)$. In fact, the antioxidant activity in vitro of this compound is higher than that of its derivative oleuropein by almost double [15]. Extracts richer in hydroxytyrosol than in oleuropein have also revealed a higher ability to attenuate the formation of advanced glycation end products, which are associated with chronic diseases and the aging process [16]. Moreover, extracts rich in oleuropein and hydroxytyrosol possessed hypolipidemic and hepatoprotective properties, but it was mainly the hydroxytyrosol-rich extract that enhanced the antioxidant status and attenuated the liver inflammation and apoptosis in rats treated with a high-fat diet [17].

Furthermore, triterpenic acids were also detected in all the extracts. The content of maslinic acid and oleanolic acid ranged between 1.8 and 5.7, and 0.4 and $1.5 \mathrm{~g} / \mathrm{kg}$ dry weight, respectively, and it shows that multifunctional extracts can be obtained, since these compounds can provide, for example, cardioprotective properties [18]. Extracts richer in these compounds can probably be produced by modulating the polarity of the solvent [19].
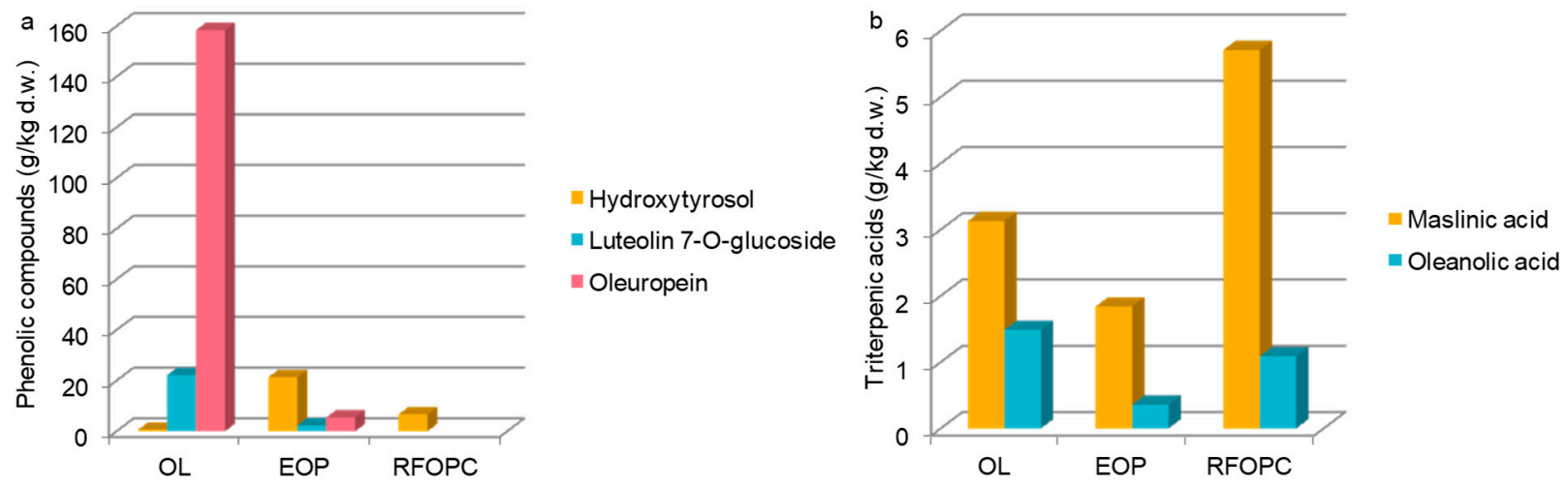

Figure 3. Content ( $\mathrm{g} / \mathrm{kg}$ dry weight) of selected phenolic compounds (a) and triterpenic acids (b) in the extracts of olive leaves (OL), exhausted olive pomace (EOP) and residual fraction from olive pits cleaning (RFOPC).

Finally, the analysis by RP-HPLC-QTOF-MS and -MS/MS confirmed the presence of these bioactive compounds in the extracts. Moreover, it revealed the presence of other phenolic compounds in the extracts, including six novel trilignols in RFOPC, which could contribute to the antioxidant properties of the extracts.

\section{Conclusions}

Besides OL, the waste products derived from olive pomace, EOP and RFOPC, are promising sources of added-value phenolic compounds with antioxidant activity. Among them, EOP contained the highest amount of hydroxytyrosol, which is also considered one of the most powerful antioxidants. Nonetheless, the extracts also presented triterpenic acids and other phenolic compounds whose characterization is of merit since they can contribute to the functional properties of the extracts.

Author Contributions: Conceptualization, M.D.M.C. and E.C.; methodology, M.D.M.C.; software, M.D.M.C.; validation, M.D.M.C. and I.G.-C.; investigation, M.D.M.C.; writing-original draft preparation, M.D.M.C.; writing-review and editing, I.G.-C., I.R., E.C.; supervision, E.C.; project administration, I.R. and M.D.M.C.; funding acquisition, I.R. and M.D.M.C. All authors have read and agreed to the published version of the manuscript. 
Funding: Financial support from “Agencia Estatal de Investigación and Fondo Europeo de Desarrollo Regional”. Reference project ENE2017-85819-C2-1-R. Authors also thank the FEDER UJA project 1260905 funded by "Programa Operativo FEDER 2014-2020" and “Consejería de Economía y Conocimiento de la Junta de Andalucía" and the UJA grant R5/04/2017.

Institutional Review Board Statement: Not applicable.

Informed Consent Statement: Not applicable.

Data Availability Statement: Data are available on request from the corresponding author.

Acknowledgments: The technical and human support provided by UJA CICT (MINECO, Junta de Andalucía, FEDER) is gratefully acknowledged.

Conflicts of Interest: The authors declare no conflict of interest.

\section{References}

1. Talhaoui, N.; Gómez-Caravaca, A.M.; León, L.; De La Rosa, R.; Fernández-Gutiérrez, A.; Segura-Carretero, A. Determination of phenolic compounds of 'Sikitita' olive leaves by HPLC-DAD-TOF-MS. Comparison with its parents 'Arbequina' and 'Picual' olive leaves. LWT-Food Sci. Technol. 2014, 58, 28-34.

2. Talhaoui, N.; Gómez-Caravaca, A.M.; León, L.; De La Rosa, R.; Fernández-Gutiérrez, A.; Segura-Carretero, A. From olive fruits to olive oil: Phenolic compound transfer in six different olive cultivars grown under the same agronomical condition. Int. J. Mol. Sci. 2016, 17, 337.

3. Contreras, M.d.M.; Romero, I.; Moya, M.; Castro, E. Olive-derived biomass as a renewable source of value-added products. Process Biochem. 2020, 97, 43-56.

4. Talhaoui, N.; Taamalli, A.; Gómez-Caravaca, A.M.; Fernández-Gutiérrez, A.; Segura-Carretero, A. Phenolic compounds in olive leaves: Analytical determination, biotic and abiotic influence, and health benefits. Food Res. Int. 2015, 77, 92-108.

5. Lopez-Huertas, E.; Fonolla, J. Hydroxytyrosol supplementation increases vitamin C levels in vivo. A human volunteer trial. Redox Biol. 2017, 11, 384-389.

6. Carnevale, R.; Silvestri, R.; Loffredo, L.; Novo, M.; Cammisotto, V.; Castellani, V.; Bartimoccia, S.; Nocella, C.; Violi, F. Oleuropein, a component of extra virgin olive oil, lowers postprandial glycaemia in healthy subjects. Br. J. Clin. Pharmacol. 2018, 84, 1566-1574.

7. Echeverría, F.; Ortiz, M.; Valenzuela, R.; Videla, L.A. Hydroxytyrosol and cytoprotection: A Projection for Clinical Interventions. Int. J. Mol. Sci. 2017, 18, 930.

8. Medfai, W.; Contreras, M.d.M.; Lama-Muñoz, A.; Mhamdi, R.; Oueslati, I.; Castro, E. How cultivar and extraction conditions affect antioxidants type and extractability for olive leaves valorization, ACS Sustain. Chem. Eng. 2020, 8, 5107-5118.

9. Contreras, Md.M.; Lama-Muñoz, A.; Gutiérrez-Pérez, J.M.; Espínola, F.; Moya, M.; Romero, I.; Castro, E. Integrated process for sequential extraction of bioactive phenolic compounds and proteins from mill and field olive leaves and effects on the lignocellulosic profile. Foods 2019, 8, 531.

10. Ali Mami, M.; Mätzing, H.; Gehrmann, H.J.; Stapf, D.; Bolduan, R., Lajili, M. Investigation of the olive mill solid wastes pellets combustion in a counter-current fixed bed reactor. Energies 2018, 11, 1965.

11. Sluiter, A.; Hames, B.; Ruiz, R.; Scarlata, C.; Sluiter, J.; Templeton, D. Laboratory Analytical Procedure TP-510-42623: Determination of sugars, byproducts, and degradation products in liquid fraction process samples. Natl. Renew. Energy Lab. 2006, $1-14$.

12. Contreras, M.d.M.; Lama-Muñoz, A.; Espínola, F.; Moya, M.; Romero, I.; Castro, E. Valorization of olive mill leaves through ultrasound-assisted extraction. Food Chem. 2020, 314, 126218.

13. Gómez-Cruz, I., Cara, C.; Romero, I.; Castro, E.; Gullón, B. Valorisation of exhausted olive pomace by an eco-friendly solvent extraction process of natural antioxidants. Antioxidants 2020, 9, 1010.

14. Ammar, S.; Contreras, M.d.M.; Gargouri, B.; Segura-Carretero, A.; Bouaziz, M. RP-HPLC-DAD-ESI-QTOF-MS based metabolic profiling of the potential Olea europaea by-product "wood" and its comparison with leaf counterpart. Phytochem. Anal. 2017, 28, 217-229.

15. Benavente-García, O.; Castillo, J.; Lorente, J.; Ortuño, A.; del Rio, J.A. Antioxidant activity of phenolics extracted from Olea europea L. leaves. Food Chem. 2000, 68, 457-462.

16. Navarro, M.; Morales, F.J.; Ramos, S. Olive leaf extract concentrated in hydroxytyrosol attenuates protein carbonylation and the formation of advanced glycation end products in a hepatic cell line (HepG2). Food Funct. 2017, 8, 944-953.

17. Fki, I.; Sayadi, S.; Mahmoudi, A.; Daoued, I.; Marrekchi, R.; Ghorbel, H. Comparative study on beneficial effects of hydroxytyrosol- and oleuropein-rich olive leaf extracts on high-fat diet-induced lipid metabolism disturbance and liver injury in rats. BioMed Res. Int. 2020, 2020, doi:10.1155/2020/1315202. 
18. Ning, S.; Dongli, L.; Xiaoqing, C.; Panpan, W.; Yu-Jing, L.; Ning, H.; Wen-Hua, C.; Wing-Leung, W. New applications of oleanolic acid and its derivatives as cardioprotective agents: A review of their therapeutic perspectives. Curr. Pharm. Des. 2019, 25, 3740-3750.

19. Xie, P.; Huang, L.; Zhang, C.; Deng, Y.; Wang, X.; Cheng, J. Enhanced extraction of hydroxytyrosol, maslinic acid and oleanolic acid from olive pomace: Process parameters, kinetics and thermodynamics, and greenness assessment. Food Chem. 2019, 276, 662-674. 